\title{
Status of Theory and Experiment in Hadronic Parity Violation
}

\author{
W. M. Snow \\ Department of Physics, Indiana University \\ Bloomington, IN 47401 USA \\ wsnow@indiana.edu \\ M. W. Ahmed \\ Department of Physics, North Carolina Central University \\ Durham, NC 27710 USA \\ ahmed@tunl.duke.edu \\ J. D. Bowman \\ Physics Division, Oak Ridge National Lab \\ Oak Ridge, TN 37831 USA \\ bowmanjd@ornl.gov \\ C. Crawford \\ Department of Physics, University of Kentucky \\ Lexington, KY 40506 USA \\ crawford@pa.uky.edu \\ N. Fomin \\ Department of Physics, University of Tennessee \\ Knoxville, TN 37916 USA \\ nfomin@utk.edu \\ H. Gao \\ Department of Physics, Duke University \\ Durham, NC 27710 USA \\ gao@phy.duke.edu \\ M. T. Gericke \\ Department of Physics, University of Manitoba \\ Winnipeg, MB R3T 2N2 Canada \\ Michael.Gericke@umanitoba.ca
}

This is an Open Access article published by World Scientific Publishing Company. It is distributed under the terms of the Creative Commons Attribution 3.0 (CC-BY) License. Further distribution of this work is permitted, provided the original work is properly cited. 
W. M. Snow et al.

\author{
V. Gudkov \\ Department of Physics, University of South Carolina \\ Columbia, SC 29208 USA \\ gudkov@sc.edu \\ B. R. Holstein \\ Department of Physics, University of Massachusetts \\ Amherst, MA 01003 USA \\ Holstein@physics.umass.edu \\ C. R. Howell \\ Department of Physics, Duke University \\ Durham, NC 27710 USA \\ howell@tunl.duke.edu \\ P. Huffman \\ Department of Physics, North Carolina State University \\ Raleigh, NC 27607 USA \\ paulhuffman@ncsu.edu \\ W. T. H. van Oers \\ TRIUMF and Department of Physics, University of Manitoba \\ Winnipeg, MB R3T 2N2 Canada \\ vanoers@triumf.ca \\ S. Penttilä \\ Physics Division, Oak Ridge National Lab \\ Oak Ridge, TN 37831 USA \\ penttilasi@ornl.gov \\ Y. K. Wu \\ Department of Physics, Duke University \\ Durham, NC 27710 USA \\ wu@fel.duke.edu
}

Published 29 February 2016

Hadronic parity violation uses quark-quark weak interactions to probe nonperturbative strong interaction dynamics through two nonperturbative QCD scales: $\Lambda_{Q C D}$ and the fine-tuned $\mathrm{MeV}$ scales of $\mathrm{NN}$ bound states in low energy nuclear physics. The current and projected availability of high-intensity neutron and photon sources coupled with ongoing experiments and continuing developments in theoretical methods provide the opportunity to greatly expand our understanding of hadronic parity violation in fewnucleon systems. The current status of these efforts and future plans are discussed.

Keywords: Parity violation; QCD; lattice gauge theory; effective field theory.

PACS numbers: 21.30.Cb, 11.30.Er, 24.70.+s, 13.75.Cs 


\section{Introduction}

Hadronic parity violation offers a window into one of the least understood sectors of the Standard Model, the neutral-current nonleptonic weak interactions. Weak interactions are well-understood at the quark level, and neutral-current contributions are highly suppressed in flavor-changing hadronic decays due to the absence of tree level flavor changing neutral currents in the Standard Model. The only experimentally accessible approach is thus to study the parity-violating (PV) component of nucleon-nucleon (NN) interactions and its manifestation in nuclear systems.

Hadronic parity-violation is also a unique probe of nonperturbative dynamics of the strong interactions at low energies. With the weak interactions known at the quark level, their manifestation in PV NN interactions is the result of an interference with the nonperturbative strong effects that confine the quarks in the nucleons and lead to the residual strong NN interaction. The large mass of the weak gauge bosons implies that the range of the weak interactions $\left(1 / M_{W, Z} \approx 0.002 \mathrm{fm}\right)$ is very small compared to the size of the nucleon (roughly $1 \mathrm{fm}$ ). The weak NN interactions are therefore sensitive to short-distance correlations between quarks inside the nucleon, and their manifestations at the hadronic level provide information about these correlations without the need of an external probe.

For low-energy nuclear reactions, hadronic parity violation can be described in terms of interactions between nucleons. The complex interplay between weak and nonperturbative strong physics is encoded in five PV NN amplitudes. Experimental observables are linear combinations of the NN amplitudes, and the relative weightings of these NN amplitudes must be calculated to map out the PV landscape with an appropriate suite of measurements. This information should be derived from two- and few-nucleon systems to avoid the still-unknown complexities of nuclear structure.

Not enough experimental information from two- and few-nucleon systems is available yet to reliably determine all of the weak NN amplitudes. However, the existing and projected availability of high-intensity sources of neutrons and photons combined with ongoing technical improvements in the control of systematic uncertainties make new experiments possible. In parallel, the theoretical tools needed for a reliable and systematic analysis and interpretation of the experimental results continue to be developed. In particular, effective field theory (EFT) methods that are applicable to few-nucleon systems provide model-independent results which by construction obey the known symmetries of QCD along with theoretically justifiable error estimates.

Ultimately, the weak NN amplitudes have to be related to Standard Model parameters by calculations at the quark level. They provide a qualitatively distinct piece of low-energy data that any nonperturbative QCD calculation has to reproduce. The relative size of the various amplitudes can provide a hint at the underlying dynamics. The first lattice QCD calculation of a hadronic parity violation amplitude appeared recently. ${ }^{1}$ The importance of lattice QCD calculations in determining PV 
nucleon interactions was also highlighted in the 2009 workshop "Forefront Questions in Nuclear Science and the Role of Computing at the Extreme Scale" .2 Since little is known about the PV nucleon couplings, they provide an opportunity to utilize lattice QCD and EFT to make predictions in the nonperturbative regime, which in turn can be experimentally tested.

\section{Current Experimental Status}

After a decade in which there was almost no new experimental information on NN weak interaction amplitudes, we now anticipate three experimental results on NN parity violation in two and few-nucleon systems which promise to greatly improve our knowledge of this sector. A 2011 NSAC review of the subfield of fundamental neutron physics identified NPDGamma as the highest priority experiment in NN weak interaction physics. NPDGamma was selected as the first experiment to run on the new FnPB facility for neutron physics at the Spallation Neutron Source at Oak Ridge National Lab. NPDGamma measures the parity-odd asymmetry of the 2.2 MeV photons from polarized slow neutron capture on protons and is sensitive to a S-P NN weak transition amplitude in the $\Delta I=1$ channel corresponding to weak pion exchange. NPDGamma finished taking data in mid-2014 and data analysis is in progress. The preliminary result for the parity-violating asymmetry $A_{\gamma}$ is that it is small with a statistical error of $\sim 13 \mathrm{ppb}$ and with negligible systematic error. ${ }^{3}$ This result is consistent with both the longstanding result from experiments and theoretical analysis of parity violation in ${ }^{18} \mathrm{~F}$, which sees no evidence for a $\Delta I=1 \mathrm{NN}$ weak amplitude from pion exchange, with a recent pioneering lattice calculation, ${ }^{1}$ and with $1 / N_{c}$ arguments. ${ }^{4,5}$

This result will have a number of implications. For the first time, the size of the S-P NN weak transition amplitude in the $\Delta I=1$ channel corresponding to weak pion exchange will soon be tightly constrained with data from a two-nucleon system. The size of this amplitude is small compared to the size of the $\Delta I=0 \mathrm{NN}$ weak amplitudes which have been constrained by previous experimental observations of parity violation in proton scattering. The four-quark operators responsible for NN weak interactions in $\Delta I=0$ and $\Delta I=1$ channels have similar strength at the electroweak scale, and the perturbative QCD evolution of these operators from the electroweak scale down to $\Lambda_{Q C D}$ does not change this. We therefore suspect that this large difference is a sign of interesting nonperturbative QCD dynamics. We also expect that the $\Delta I=1 \mathrm{NN}$ weak amplitudes are dominated by quark-quark neutral currents as the charged current contributions at the electroweak scale are suppressed by a factor of $V_{u s}^{2}$. If so, the NPDGamma result implies that quark-quark neutral currents seem to be suppressed in NN weak transition amplitudes.

The long range of the $\Delta I=1 \mathrm{NN}$ weak amplitude corresponding to weak pion exchange contributes to almost all parity-odd observables in two- and few-nucleon systems. The NPDGamma result that this amplitude is both small and tightly constrained will make it much easier to extract new information on the other NN 
weak transition amplitudes from additional experiments in two- and few-nucleon systems.

Important progress has been made on two other parity violation experiments in few-nucleon systems. An experiment to measure the proton asymmetry in polarized slow neutron capture on ${ }^{3} \mathrm{He}$ (the $\overrightarrow{s_{n}} \cdot \vec{k}_{p}$ correlation in $\vec{n}+{ }^{3} \mathrm{He} \rightarrow{ }^{3} \mathrm{H}+p$ ) is taking data on the FnPB at the SNS with a projected statistical accuracy of $1.6 \times 10^{-8} \cdot 6,7$ The experiment has measured a small ( ppm) parity-conserving asymmetry $\left(\overrightarrow{s_{n}} \cdot\left(\overrightarrow{k_{n}} \times \overrightarrow{k_{p}}\right)\right)$ correlation. The predicted PV asymmetry is $1.2 \times 10^{-7}$. A statistically-limited null result in polarized slow neutron spin rotation in $\vec{n}+{ }^{4} \mathrm{He}$ was measured on the NG-6 beamline at $\mathrm{NIST}^{8}{ }^{8}$ and a new spin rotation apparatus is under construction which is proposed to run on the new more intense NG-C beam at NIST. The projected precision of both of these experiments is enough to tightly constrain linear combinations of parity-odd transition amplitudes. Also, in both of these parity-odd observables the relative signs of the $\Delta I=0$ and $\Delta I=1$ transition amplitudes are different from the isospin-conjugate $\vec{p}+{ }^{4} \mathrm{He}$ system, where a nonzero parity-odd asymmetry was measured earlier at PSI. Data on parity violation in proton-proton scattering already exists as well. We therefore anticipate that the addition of the new results from these three experiments should greatly reduce the uncertainly on the values of these parity-odd amplitudes.

\section{Future Experimental Opportunities}

After these three experiments are completed over the next few years, we will have a much better feeling for what should be the highest priority for future experiments. The NPDGamma experiment is not limited by systematic errors, and it is anticipated that the same might well be true for the $n-{ }^{3} \mathrm{He}$ and $n+{ }^{4} \mathrm{He}$ measurements. It is therefore likely that it would be interesting to repeat one or more of these experiments should a higher intensity slow neutron beamline become available. A very interesting slow neutron beamline for experiments of relevance to nuclear/particle/astrophysics/cosmology has recently been proposed for the European Spallation Source (ESS) now under construction in Lund, Sweden. The ESS is designed as a so-called "long-pulse" spallation neutron source with a design power of $5 \mathrm{MW}$, larger than the SNS by a factor of 5 . Combined with some improvements in the brightness in slow neutron moderators, it promises to deliver more than an order of magnitude more polarized slow neutrons than any other pulsed neutron source. The pulsed nature of the source is very important for these experiments to successfully isolate systematic errors as one gets neutron energy information from time-of-flight and many possible systematic effects depend on the neutron kinetic energy. If this beamline is realized it will be possible to either improve the statistical accuracy of one or more of the experiments mentioned above or perhaps to measure NN parity violation in a different system such as polarized slow neutron capture on deuterium. By the time that this facility becomes available we will be able to make a much more intelligent choice of what experiment to pursue given 
the results of the ongoing experiments combined with the ongoing progress from theory.

There is another interesting scientific opportunity in experimental NN weak interaction physics. This is the measurement of the parity-odd helicity dependence of the photodisintegration of the deuteron near threshold in the $\vec{\gamma}+D \rightarrow n+p$ reaction. Together with the parity-odd longitudinal asymmetry in $\vec{p}+p$ scattering, this observable is sensitive to the experimentally elusive $\Delta I=2 \mathrm{NN}$ weak amplitude. This amplitude is especially interesting from a theoretical point of view as it comes from one $\Delta I=2$ effective 4-quark operator above $\Lambda_{Q C D}$ and it is the most accessible channel for a calculation from the Standard Model using lattice gauge theory. This experiment could be done in the future at a high-intensity polarized gamma source. The proposed upgrades to the $\mathrm{HI} \gamma \mathrm{S}$ facility at Duke in beam intensity and in the capability for rapid and controlled helicity flipping of the photon beam could make it possible to conduct this experiment. Such an upgrade could also enable other parity violation experiments in few nucleon systems.

\section{Theoretical Status}

Traditionally PV NN interactions have been described in a meson-exchange picture in analogy to many parity-conserving (PC) NN models. Most widely used has been the DDH formulation, ${ }^{9}$ which also provides estimated ranges for the PV couplings appearing in the model. The available experimental results from two- to manynucleon systems can be described in terms of four DDH parameters, provided a one-body PV potential is derived in the case of heavy nuclei. ${ }^{10}$ Only one $\Delta I=1$ parameter is needed to describe the experimental data.

Over the last decades, EFTs have emerged as alternatives to phenomenological models of the NN interactions, both in the PC and PV sectors. EFTs are model independent, and provide systematic theoretical error estimates and a connection to QCD via symmetry principles. The use of EFTs to study hadronic parity violation goes back to the $90 \mathrm{~s},{ }^{11}$ with a comprehensive analysis of PV Lagrangians given in Ref. 12. EFTs have now been applied to PV observables in two-, three-, and four-nucleon systems (see Ref. 13 for a recent review), including the corresponding observables discussed in the previous sections. Such calculations play an important role in assessing whether measurements are feasible and, if so, in the planning and the subsequent analysis of the corresponding experiments. EFT methods continue to be developed, with a particular emphasis on the application to few-nucleon systems. $p+{ }^{4}$ He scattering at low energy was recently calculated using chiral EFT potentials. ${ }^{14} \mathrm{PV}$ three-nucleon interactions were shown to be $10 \%$ effects. ${ }^{15}$ This means that at very low energy the five PV low-energy constants (LECs) are sufficient to describe parity violation in few-nucleon systems. The PV asymmetry in $\vec{n}+{ }^{3} \mathrm{He} \rightarrow{ }^{3} \mathrm{H}+p$ was calculated employing chiral EFT for both $\mathrm{PC}$ and $\mathrm{PV}$ interactions. This calculation is essential for the interpretation of the upcoming measurement of this observable at the FnPB. The relationships and connections 
between two different descriptions of $\mathrm{NN}$ weak interactions, the model-dependent $\mathrm{DDH}$ approach and the various versions of EFT have been greatly clarified onver the last few years.

There has been tremendous progress in the development of lattice QCD as a reliable tool to compute basic properties of low-energy nuclear and hadronic physics. Lattice QCD is now being utilized to make theoretical predictions of important low-energy strong interaction amplitudes which are either challenging or currently not possible to measure experimentally. The pioneering calculation of the PV pionnucleon coupling in Ref. 1 showed that lattice QCD can have significant impact on our understanding of hadronic parity violation. The resulting value of $h_{\pi}^{1 \text {,con }}=$ $\left(1.099 \pm 0.505_{-0.064}^{+0.058}\right) \times 10^{-7}$ is in agreement with present experimental bounds. It is possible to utilize lattice QCD to make predictions of the yet undetermined PV coupling constants appearing in the PV EFT, which in turn can be tested experimentally. This program could establish a quantitative connection through two nonperturbative strong interaction scales $\left(\Lambda_{Q C D}\right.$ and the $\sim \mathrm{MeV}$ scale of $\mathrm{NN}$ bound state dynamics) from quark-level interactions to observables in few-nucleon systems. The demonstration of success in this very nontrivial goal would represent an impressive milestone in our understanding of the strongly interacting limit of QCD.

\section{Theoretical Opportunities}

There are a number of opportunities for theoretical progress in hadronic parity violation. The ongoing improvements in the application of EFT methods to few-nucleon systems put consistent calculations of the PV asymmetry in $\vec{p}+{ }^{4} \mathrm{He}$ (which has been measured) and the spin rotation angle in $\vec{n}+{ }^{4} \mathrm{He}$ (which is planned for NIST) within reach. The power counting analysis of EFTs is based on the assumption that the couplings are natural in size. Experimental results that reveal a pattern of values for the LECs different from $\mathrm{O}(1)$ are an indication of nontrivial QCD dynamics. An updated analysis of the EFT power counting may be required in that case. Lattice QCD plays an equally important role in establishing the connection between the fundamental Standard Model parameters to weak NN amplitudes. The $\Delta I=2$ amplitude presents an especially interesting target for investigation because it only receives contributions from connected quark diagrams (not the computationally demanding disconnected loop diagrams) and the isotensor nature of the operator prevents mixing under renormalization. First lattice QCD results on this amplitude from the CalLat Collaboration are anticipated in the near future. The determination of this quantity from experiment is difficult and currently not well constrained. Our ignorance of the size of this $\Delta I=2$ amplitude also contributes significantly to the uncertainty in the determination of the $\Delta I=0$ amplitude from p-p scattrering. ${ }^{16}$ This amplitude presents a very exciting opportunity to use lattice QCD and EFT to make a precise prediction of a NN weak amplitude directly from the Standard Model. 


\section{HI $\gamma$ S2: The Compton $\gamma$-Ray Source}

The measurement of the parity-violating asymmetry of photodisintegration of the deuteron to an accuracy of $10^{-8}$ requires a high intensity circularly polarized $\gamma$-ray beam with high polarization $(>90 \%)$ and narrow beam energy spread $(<100 \mathrm{keV}$ FWHM at about 2.4 MeV). The current facility best suited to deliver the $\gamma$-ray beam for this experiment is the High Intensity Gamma-ray Source $(\mathrm{HI} \gamma \mathrm{S})$ at the Triangle Universities Nuclear Laboratory (TUNL). The $\gamma$-ray beam at $\mathrm{HI} \gamma \mathrm{S}$ is produced by Compton backscattering of the photons inside the optical cavity of a storage-ring based Free-Electron Laser from circulating electron bunches. The current capabilities of $\mathrm{HI} \gamma \mathrm{S}$ are: (1) delivery of $\gamma$-ray beams on target in the energy of 1 to 100 $\mathrm{MeV}$ with beam energy spread selected by collimation as low as about $1 \%$ FWHM, (2) a beam intensity on target with $5 \%$ energy spread as high as $10^{9}$ gammas $/ \mathrm{s}$, (3) linear or circular beam polarization with magnitude of polarization greater than $95 \%$. To carry out the proposed parity violation experiment will require an upgrade of $\mathrm{HI} \gamma \mathrm{S}$ to increase the beam intensity by about two orders of magnitude and to provide the capability of fast reversal of the beam helicity with precision control and diagnostics of the spatial and energy distributions of the beam. A two-stage upgrade of $\mathrm{HI} \gamma \mathrm{S}$ is envisaged. The first stage is an upgrade of the electron injector system to increase the average charge injection rate into the storage ring and to enhance the overall reliability of the accelerator drivers. This stage is motivated by the Compton-scattering research program at $\mathrm{HI} \gamma \mathrm{S}$ and is also needed for the parity-violation experiments. This part of the accelerator upgrade will start around 2018. The second stage in the upgrade is the installation of an optical cavity in the straight section of the storage ring that is driven by an external laser. The photons in the optical cavity will produce $\gamma$-rays by Compton scattering from the electron bunches circulating in the storage ring. The schedule for starting construction is around 2021.

A schematic diagram of $\mathrm{HI} \gamma \mathrm{S} 2$ is shown in Fig. 1. The $\mathrm{HI} \gamma \mathrm{S} 2 \gamma$-ray beam is produced by colliding a high-current electron beam with photons inside an optical cavity that is driven by a powerful external laser. The electron beam is provided by the storage ring which will be operated with 32 electron bunches with a total current up to about $500 \mathrm{~mA}$. An average optical power of about 10 to $20 \mathrm{KW}$ will be built up inside the high-finesse Fabry-Perot resonator. Compared with the FEL driven $\mathrm{HI} \gamma \mathrm{S}$, a higher average intracavity laser power and significantly reduced beam sizes at the collision point make it possible for the $\mathrm{HI} \gamma \mathrm{S} 2$ to achieve a total gamma flux of $10^{11}$ to $10^{12} \mathrm{\gamma} / \mathrm{s}$ in the energy range of 2 to $12 \mathrm{MeV}$. In addition to the substantial intensity increase, the $\mathrm{HI} \gamma \mathrm{S} 2$ facility will produce $\gamma$-ray beams with a better monochromaticity and the ability to rapidly switch the $\gamma$-ray beam helicity at a rate of tens of $\mathrm{Hz}$ or higher. The fast helicity switch of the $\gamma$-ray beam will be realized by changing the polarization of the laser beam outside the Fabry-Perot resonator using polarizing optics such as Pockels cells and wave plates. 


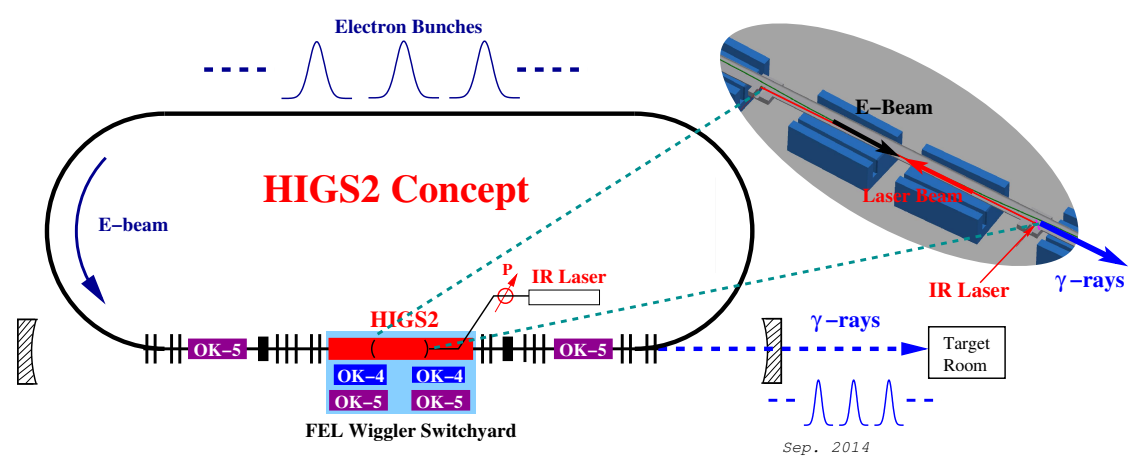

Fig. 1. The schematic layout of the HI $\gamma \mathrm{S} 2$ Compton $\gamma$-ray source. The HI $\gamma \mathrm{S} 2$ is located in one of the two long straight sections of the electron storage ring. Guided by a set of deflection magnets, the electron beam enters and exits the high-power Fabry-Perot resonator to collider with the photon pulse in the center of the resonator. The polarization of the $\gamma$-ray beam is rapidly switchable by changing the polarization of the laser beam from the external infrared drive laser using polarizing optics.

\section{Summary and Conclusions}

Quark-quark weak interactions in the Standard Model induce parity-odd nucleonnucleon interactions. Hadronic parity violation provides a unique probe of neutralcurrent nonleptonic weak interactions as well as of nonperturbative strong dynamics. The preliminary result from the NPDGamma experiment at ORNL, the first new sensitive experimental result in the NN system in many years, shows that at least one NN weak amplitude is smaller than expected on symmetry arguments alone. It strongly supports the longstanding suspicion from analysis of previous data that NN weak amplitudes are sensitive to poorly-understood aspects of nonperturbative QCD dynamics. This observation is intellectually exciting as it shows that NN weak interaction measurements could open a unique window on nonperturbative QCD physics. The availability of high-intensity sources of neutrons and photons such as the FnPB at the Spallation Neutron Source at ORNL, the new NG-C neutron beam at the NCNR at NIST, the European Spallation Source under construction in Sweden, and an upgraded HI $\gamma$ S gamma facility at TUNL along with the controls for systematic uncertainties possible at these facilities provides new opportunities to greatly improve our experimental information on hadronic parity violation. Effective field theory methods are ideally suited to analyze and interpret the experimental results in this field. The recent progress in the application of effective field theories to few-nucleon systems should make it possible to determine PV observables in processes involving as many as five nucleons. The weak amplitudes at the nucleon level should ultimately be connected to the parameters of the Standard Model. An understanding of the neutral-current weak interaction and its manifestation in 
hadronic parity violation from the Standard Model will require a coordinated effort between experiment, theory and computational physics.

\section{Acknowledgments}

We gratefully acknowledge the extensive input of M. R. Schindler on this document. WMS acknowledges support through the Indiana University Center for Spacetime Symmetries.

\section{References}

1. J. Wasem, Phys. Rev. C 85, 022501 (2012).

2. Scientific Grand Challenges: Forefront Questions in Nuclear Science and the Role of Computing at the Extreme Scale, in Workshop sponsored by the DOE Office of Nuclear Physics and the Office of Advanced Scientific Computing, 2009.

3. J. D. Bowman, presentation at the Electron-Nucleus Scattering XIII conference, Elba, Italy. June 2014.

4. N. Kaiser and U. G. Meissner, Nucl. Phys. A 499, 699 (1989).

5. D. R. Phillips, D. Samart, and C. Schat, Phys. Rev. Lett. 99, (2014).

6. V. Gudkov, Phys. Rev. C 82, 065502 (2010).

7. M. Vivani et al, Phys. Rev. C 89, 064004 (2014).

8. W. M. Snow et al., Phys.Rev. C 83, 022501 (2011).

9. B. Desplanques, J. F. Donoghue, and B. R. Holstein, Annals Phys. (NY) 124, 449 (1980).

10. J. D. Bowman, presentation at INT program on "Electric Dipole Moments and CP Violations", (2007).

11. D. B. Kaplan and M. J. Savage, Nucl. Phys. A 556, 653 (1993).

12. S. L. Zhu, C. M. Maekawa, B. R. Holstein, and M. J. Ramsey-Musolf, Nucl. Phys. A 748, 435 (2005).

13. M. R. Schindler and R. P. Springer, Prog. Part. Nucl. Phys. 72, 1 (2013).

14. G. Hupin, S. Quaglioni, and P. Navratil, Phys. Rev. C 90, 061601 (2014).

15. H. W. Griesshammer and M. R. Schindler, Eur. Phys. J A 46, 73 (2010).

16. W. C. Haxton and B. Holstein, Prog. Part. Nucl. Phys. 71, 185 (2013). 